Annuaire suisse de politique de développement

$21 \mid 2002$

Agriculture suisse et mondialisation

\title{
2. Flux financiers
}

\section{Gérard Perroulaz}

\section{OpenEdition}

Journals

Édition électronique

URL : http://journals.openedition.org/aspd/953

DOI : 10.4000/aspd.953

ISSN : 1663-9669

\section{Éditeur}

Institut de hautes études internationales et du développement

\section{Édition imprimée}

Date de publication : 1 avril 2002

Pagination : 333-348

ISSN : 1660-5934

\section{Référence électronique}

Gérard Perroulaz, «2. Flux financiers », Annuaire suisse de politique de développement [En ligne], 21 |

2002, mis en ligne le 07 septembre 2012, consulté le 08 septembre 2020. URL : http://

journals.openedition.org/aspd/953; DOI : https://doi.org/10.4000/aspd.953 


\section{FLUX FINANCIERS*}

\subsection{Aperçu général des flux financiers destinés aux pays en développement}

\section{A. Flux nets en provenance de la Suisse $(1998,1999$ et 2000)}

\begin{tabular}{|c|c|c|c|c|c|c|}
\hline \multirow{2}{*}{ Catégories } & 1998 & 1999 & $2000^{p}$ & 1998 & 1999 & $2000^{p}$ \\
\hline & \multicolumn{3}{|c|}{ En millions de francs } & \multicolumn{3}{|c|}{ En \% du RNB } \\
\hline 1. Capitaux privés ${ }^{1}$ & 5194,3 & 3360,0 & 1680,2 & 1,28 & 0,80 & 0,39 \\
\hline dont: Investissements directs & 5194,3 & 2756,0 & 1912,9 & & & \\
\hline Crédits à l'exportation & n.d. & 604,1 & 843,5 & & & \\
\hline Investissements de portefeuille & n.d. & n.d. & 1076,2 & & & \\
\hline 2. Aide publique au développement ${ }^{2}$ & 1301,8 & 1478,7 & 1501,3 & 0,32 & 0,35 & 0,34 \\
\hline 3. Autres apports publics ${ }^{3}$ & 50,7 & 31,6 & 13,5 & 0,01 & 0,01 & 0,00 \\
\hline $\begin{array}{l}\text { 4. Dons des organismes } \\
\text { privés bénévoles }{ }^{4}\end{array}$ & 249,4 & 275,4 & 274,1 & 0,06 & 0,07 & 0,06 \\
\hline Total & 6796,3 & 5145,7 & 3469,1 & & & \\
\hline \multicolumn{7}{|l|}{ dont flux assortis de: } \\
\hline conditions du marché $(=1+3)$ & 5245,0 & 3391,6 & 1693,6 & 1,29 & 0,81 & 0,39 \\
\hline conditions libérales $(=2+4)$ & 1551,3 & 1754,1 & 1456,1 & 0,38 & 0,42 & 0,33 \\
\hline RNB de la Suisse & 405916,0 & 417750,6 & 435220,2 & & & \\
\hline
\end{tabular}

Source des tableaux 2.1.: OCDE, Coopération pour le développement, Rapport 2001 Développement international. OCDE, Les dossiers du CAD, vol. 3, n 1, Paris, 2002, tableaux 12-13.

RNB = Revenu national brut.

${ }^{\mathrm{P}}$ chiffres provisoires.

n.d. : données non disponibles.

Notes 1 à 4 : voir page suivante.

* Par Gérard Perroulaz. 
Note: les catégories de flux financiers figurant dans le tableau ont été définies par le Comité d'aide au développement de l'OCDE.

1. Les flux de capitaux privés sont des apports de ressources aux conditions du marché provenant de l'économie privée.

- Investissements directs: investissements effectués pour acquérir ou augmenter des intérêts durable dans une entreprise d'un autre pays. Les flux d'investissements directs correspondent à la variation de l'actif net que les filiales représentent dans la comptabilité de la société-mère. Ils ne sont pas forcément accompagnés de transferts de capitaux au sens de la définition de la balance des paiements donnée par le Fonds monétaire international. La statistique est établie sur la base des données communiquées par les entreprises.

口 Les crédits à l'exportation sont des prêts, accordés pour une durée de plus d'un an pour l'achat de marchandises dans le pays créancier. Les montants indiqués dans le tableau concernent seulement les prêts garantis.

- Les investissements de portefeuille comprennent deux types de flux :

a) Les apports bilatéraux : souscription d'obligations émises par des Etats du Tiers Monde ou par des sociétés établies dans des pays en développement, acceptation de reconnaissance de dettes, achat d'actions, d'obligations et de biens immobiliers.

b) Les apports multilatéraux: investissements du secteur privé bancaire et non bancaire dans des titres émis par des institutions multilatérales.

- Les flux bancaires, qui représentent la variation annuelle des avoirs nets détenus par les banques résidantes (y compris les comptoirs à l'étranger) vis-à-vis des pays en développement, ne sont pas comptés.

2. L'aide publique au développement (APD) représente l'ensemble des flux financiers accordés aux pays et territoires figurant dans la partie 1 de la liste des pays bénéficiaires de l'aide établie par le CAD (pays en développement). L'APD comprend aussi les contributions aux institutions multilatérales de financement provenant de fonds publics (y compris, en ce qui concerne la Suisse, les prestations des cantons et des communes). Ces prestations sont en principe fournies dans le but essentiel de favoriser le développement économique des pays en développement et elles sont accordées à des conditions de faveur. Pour les composantes de l'APD, voir les tableaux de la section 3 .

Le chiffre d'aide publique au développement fourni par la Suisse figurant dans les statistiques du CAD peut être différent de celui qui figure dans les statistiques suisses, ceci pour deux raisons:

a) la Confédération s'acquitte de certaines contributions internationales par l'émission de notes dont l'encaissement intervient ultérieurement. Ces engagements sont compris dans le chiffre de l'aide déclaré à l'OCDE au moment de l'engagement, alors que la Confédération publie un montant d'aide sur la base des versements effectifs de l'année.

b) Des différences dans le montant de l'APD de la Suisse peuvent aussi provenir de révisions des données statistiques pendant l'année. Les sources de certains tableaux peuvent dater de juin 2001 (Rapport annuel de la coopération internationale) ou de chiffres communiqués par la DDC ultérieurement (octobre 2001 ou février 2002). Ceci explique que certains chiffres ont été révisés.

3. Les autres apports du secteur public comprennent tous les autres moyens mis à disposition des pays en développement par des collectivités publiques mais qui ne sont pas assortis de conditions de faveur.

4. Les dons des organismes privés bénévoles comprennent les prestations accordées par des institutions sans but lucratif (sans les subventions reçues du secteur public).

Le revenu national brut (RNB) de la Suisse est estimé aux prix (courants) du marché. 


\subsection{Aperçu général des flux financiers destinés aux pays en développement}

\section{B. Flux nets en provenance des pays membres du CAD $(1998,1999$ et 2000)}

\begin{tabular}{|c|c|c|c|c|c|c|}
\hline \multirow{2}{*}{ Catégories } & 1998 & 1999 & $2000^{p}$ & 1998 & 1999 & $2000^{p}$ \\
\hline & \multicolumn{3}{|c|}{ En millions de dollars } & \multicolumn{3}{|c|}{ En \% du RNB } \\
\hline 1. Capitaux privés ${ }^{1}$ & 111223,0 & 115119,0 & 74537,0 & 0,49 & 0,48 & 0,31 \\
\hline dont: Investissements directs & 83416,0 & 94708,0 & 67234,0 & & & \\
\hline Crédits à l'exportation & 2104,0 & 1896,0 & 7642,0 & & & \\
\hline Investissements de portefeuille & 25703,0 & 18515,0 & $-339,0$ & & & \\
\hline 2. Aide publique au développement ${ }^{2}$ & 52084,0 & 56428,0 & 53737,0 & 0,23 & 0,24 & 0,22 \\
\hline 3. Autres apports publics ${ }^{3}$ & 13491,0 & 15477,0 & $-4537,0$ & 0,06 & 0,07 & $-0,02$ \\
\hline $\begin{array}{l}\text { 4. Dons des organismes } \\
\text { privés bénévoles }{ }^{4}\end{array}$ & 5609,0 & 6715,0 & 6935,0 & 0,02 & 0,03 & 0,03 \\
\hline Total & 182407,0 & 193739,0 & 130672,0 & & & \\
\hline \multicolumn{7}{|l|}{ dont flux assortis de: } \\
\hline conditions du marché $(=1+3)$ & 124714,0 & 130596,0 & 70000,0 & 0,55 & 0,55 & 0,29 \\
\hline conditions libérales $(=2+4)$ & 57693,0 & 63143,0 & 60672,0 & 0,26 & 0,27 & 0,25 \\
\hline \multicolumn{7}{|c|}{ En milliards de dollars } \\
\hline RNB des pays du CAD & 22535,0 & 23784,0 & 24044,0 & & & \\
\hline
\end{tabular}

RNB = Revenu national brut.

${ }^{\mathrm{P}}$ chiffres provisoires.

Voir page précédente pour la description des catégories de flux financiers et les notes 1 à 4 . 


\subsection{Relations entre le revenu national brut (RNB), les flux nets de capitaux privés et l'aide publique au développement}

\section{A. Comparaisons entre les pays membres du CAD (2000)}

\begin{tabular}{|c|c|c|c|c|c|c|c|c|c|c|}
\hline \multirow{3}{*}{$\begin{array}{l}\text { Pays membres } \\
\text { du Comité d'aide } \\
\text { au développement }\end{array}$} & \multicolumn{3}{|c|}{$\begin{array}{l}\text { Revenu national brut } \\
\text { (RNB) }\end{array}$} & \multirow{2}{*}{\multicolumn{3}{|c|}{$\begin{array}{c}\begin{array}{c}\text { Flux nets de } \\
\text { capitaux privés }\end{array} \\
\text { Montants En \% du RNB }\end{array}$}} & \multicolumn{4}{|c|}{$\begin{array}{l}\text { Flux nets } \\
\text { d'APD }^{2}\end{array}$} \\
\hline & \multirow{2}{*}{$\begin{array}{c}\text { Montants } \\
\text { Mia \$ }\end{array}$} & \multicolumn{2}{|r|}{ Par habitant } & & & & \multirow{2}{*}{$\begin{array}{c}\text { Montants } \\
\text { Mio\$ }\end{array}$} & \multicolumn{3}{|c|}{ En \% du RNE } \\
\hline & & $\%$ & $\$$ & Mio \$ & $\%$ & Rang & & $\%$ & $\%$ & Rang \\
\hline Etats-Unis & 9929 & 41,3 & 36100 & 10666 & 0,11 & 16 & 9955 & 18,52 & 0,10 & 22 \\
\hline Japon & 4808 & 20,0 & 37900 & 2725 & 0,06 & 17 & 13508 & 25,14 & 0,28 & 12 \\
\hline Allemagne & 1860 & 7,7 & 22600 & 7000 & 0,38 & 11 & 5030 & 9,36 & 0,27 & 13 \\
\hline Royaume-Uni & 1418 & 5,9 & 23700 & 2093 & 0,15 & 14 & 4501 & 8,38 & 0,32 & 9 \\
\hline France & 1285 & 5,3 & 21800 & 1439 & 0,11 & 15 & 4105 & 7,64 & 0,32 & 8 \\
\hline Italie & 1074 & 4,5 & 18800 & 9537 & 0,89 & 5 & 1376 & 2,56 & 0,13 & 21 \\
\hline Canada & 684 & 2,8 & 22300 & 4621 & 0,68 & 6 & 1744 & 3,25 & 0,25 & 16 \\
\hline Espagne & 552 & 2,3 & 14000 & 22272 & 4,03 & 2 & 1195 & 2,22 & 0,22 & 19 \\
\hline Pays-Bas & 375 & 1,6 & 23500 & 3469 & 0,93 & 4 & 3135 & 5,83 & 0,84 & 2 \\
\hline Australie & 370 & 1,5 & 19300 & -219 & - & - & 987 & 1,84 & 0,27 & 14 \\
\hline Suisse & 258 & 1,1 & 35900 & 997 & 0,39 & 10 & 890 & 1,66 & 0,34 & 7 \\
\hline Belgique & 229 & 1,0 & 22400 & 1394 & 0,61 & 7 & 820 & 1,53 & 0,36 & 6 \\
\hline Suède & 225 & 0,9 & 25300 & 2127 & 0,95 & 3 & 1799 & 3,35 & 0,80 & 4 \\
\hline Autriche & 186 & 0,8 & 22900 & 560 & 0,30 & 13 & 423 & 0,79 & 0,23 & 18 \\
\hline Norvège & 158 & 0,7 & 35200 & -5 & 0,00 & 19 & 1264 & 2,35 & 0,80 & 3 \\
\hline Danemark & 157 & 0,7 & 29400 & 482 & 0,31 & 12 & 1664 & 3,10 & 1,06 & 1 \\
\hline Finlande & 119 & 0,5 & 23100 & 672 & 0,56 & 8 & 371 & 0,69 & 0,31 & 10 \\
\hline Grèce & 112 & 0,5 & 10600 & - & - & - & 226 & 0,42 & 0,20 & 20 \\
\hline Portugal & 104 & 0,4 & 10400 & 4273 & 4,11 & 1 & 271 & 0,50 & 0,26 & 15 \\
\hline Irlande & 79 & 0,3 & 20900 & 416 & 0,53 & 9 & 235 & 0,44 & 0,30 & 11 \\
\hline Nvlle-Zélande & 45 & 0,2 & 11800 & 17 & 0,04 & 18 & 113 & 0,21 & 0,25 & 17 \\
\hline Luxembourg & 18 & 0,1 & 40800 & - & - & - & 127 & 0,24 & 0,71 & 5 \\
\hline Total ou moyenne & 24045 & 100,0 & 28500 & 74536 & 0,31 & & 53739 & 100,00 & 0,22 & \\
\hline
\end{tabular}

Source: OCDE, Coopération pour le développement, Rapport 2001, Développement international. OCDE, Les dossiers du CAD, vol. 3, n 1 , Paris, 2002, tableau 13 et 37.

n.d. Chiffres non disponibles.

1. Y compris des montants affectés à l'annulation de créances commerciales. La remise de créances militaires n'est pas comptée.

2. L'APD fournie par la France comprend l'aide aux territoires d'outre-mer (TOM). Par contre, la statistique exclut les transferts aux départements d'outre-mer (DOM). 


\subsection{B.1. APD nette des pays membres du CAD en 2000}

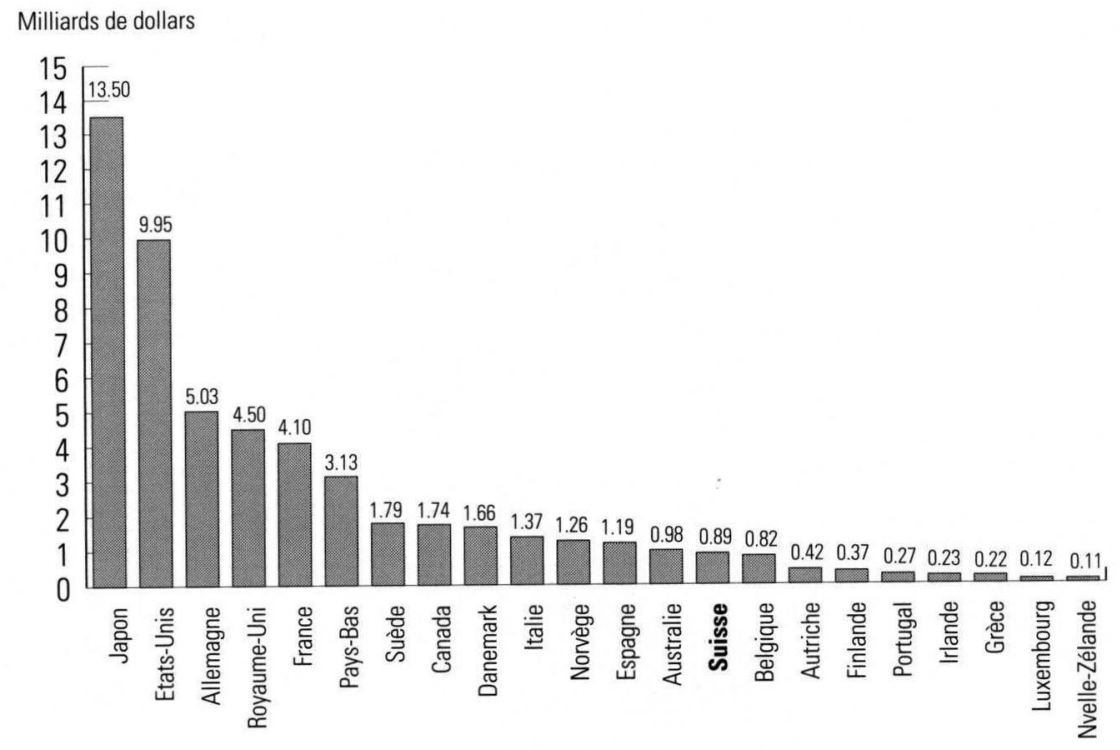

\subsection{B.2. APD nette en 2000, en pourcentage du RNB}

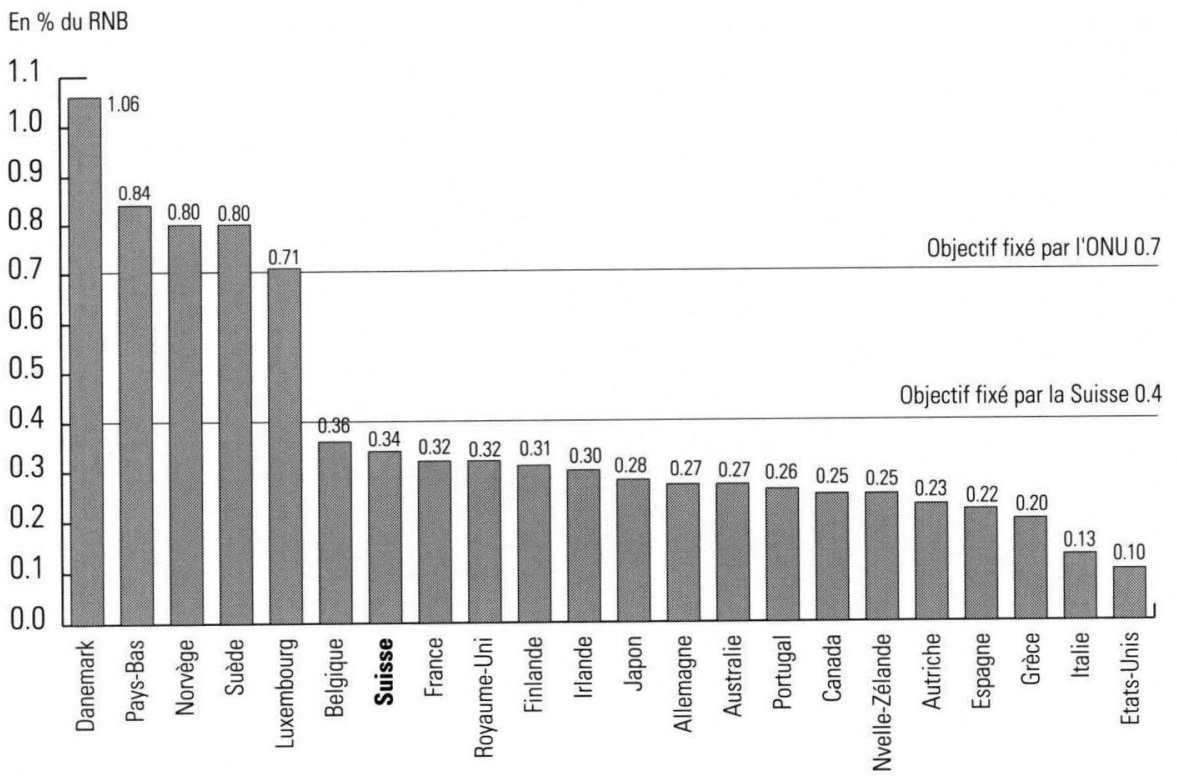

Source: OCDE, Coopération pour le développement, Rapport 2001, tableau 13. 


\subsection{Aperçu des relations financières avec le monde}

\section{A. Emprunts de débiteurs étrangers, par groupes de pays, en millions de francs ${ }^{1}$}

\section{Année 1999}

\begin{tabular}{lccc}
\hline Groupe de pays & $\begin{array}{c}\text { Valeur } \\
\text { d'émission }\end{array}$ & Remboursement & $\begin{array}{c}\text { Prélèvements nets } \\
\text { sur le marché }\end{array}$ \\
\hline Union européenne & 19845,1 & 12498,1 & 7347,0 \\
\hline Reste de l'Europe & 4717,2 & 500,0 & 4217,2 \\
\hline Etat-Unis, Canada & 5620,8 & 2640,0 & 2980,8 \\
\hline Caraïbes & 7551,3 & 700,0 & 6851,3 \\
\hline Amérique latine & & & 0,0 \\
\hline Moyen-Orient, Afrique & 370,2 & 860,0 & 0,0 \\
\hline Japon & & 250,0 & $-489,8$ \\
\hline Australie, Nouvelle-Zélande & & & $-250,0$ \\
\hline Autres pays d'Asie, Océanie & 808,0 & 1779,5 & 0,0 \\
\hline $\begin{array}{l}\text { Organisations internationales } \\
\text { de développement }\end{array}$ & $\mathbf{3 8 9 1 2 , 6}$ & $\mathbf{1 9 2 2 7 , 6}$ & $-971,5$ \\
\hline Total & & & $\mathbf{1 9 6 8 5 , 0}$ \\
\hline
\end{tabular}

\section{Année 2000}

\begin{tabular}{lrrr}
\hline Groupe de pays & $\begin{array}{c}\text { Valeur } \\
\text { d'émission }\end{array}$ & Remboursement & $\begin{array}{c}\text { Prélèvements nets } \\
\text { sur le marché }\end{array}$ \\
\hline Union européenne & 18082,7 & 12191,4 & 5891,3 \\
\hline Reste de l'Europe & 2374,5 & 2045,0 & 329,5 \\
\hline Etat-Unis, Canada & 5817,7 & 5883,0 & $-65,3$ \\
\hline Caraïbes & 6697,3 & 750,0 & 5947,3 \\
\hline Amérique latine & 0,0 & & 0,0 \\
\hline Moyen-Orient, Afrique & 0,0 & & 0,0 \\
\hline Japon & 501,8 & 2260,0 & $-1758,2$ \\
\hline Australie, Nouvelle-Zélande & 0,0 & 750,0 & $-750,0$ \\
\hline Autres pays d'Asie, Océanie & 0,0 & & 0,0 \\
\hline $\begin{array}{l}\text { Organisations internationales } \\
\text { de développement }\end{array}$ & 0,0 & 1076,4 & $-1076,4$ \\
\hline Total & $\mathbf{3 3 4 7 4 , 0}$ & $\mathbf{2 4 9 5 5 , 8}$ & $\mathbf{8 5 1 8 , 2}$ \\
\hline
\end{tabular}

Source: Banque nationale suisse, Bulletin mensuel de statistiques économiques, tableau F42, janvier 2002. Disponible sur Internet: <www.snb.ch>.

1. Emissions publiques.

2. Valeur d'émission moins remboursements.

3. Groupe de la Banque mondiale (BIRD, IDA, SFI, AMGI), Banque interaméricaine de dév. (BID), Banque africaine de développement (BAfD), Banque asiatique de développement (BAsD), Banque européenne pour la reconstruction et le développement (BERD). 


\subsection{Aperçu des relations financières avec le monde}

B. Avoirs et engagements à l'étranger des banques établies en Suisse ${ }^{1}$

1. Avoirs et engagements figurant aux bilans ${ }^{2}$

Situation au 31 décembre 2000

\begin{tabular}{|c|c|c|c|c|}
\hline \multirow[t]{2}{*}{ Pays } & $\begin{array}{c}\text { Avoirs } \\
\text { A }\end{array}$ & $\begin{array}{c}\text { Engagem. } \\
\text { B }\end{array}$ & $\begin{array}{l}\text { Solde } \\
\mathrm{C}=\mathrm{A} \cdot \mathrm{B}\end{array}$ & \multirow{2}{*}{$\begin{array}{r}\begin{array}{r}\text { Couverture } \\
\mathrm{D}=\mathrm{A} / \mathrm{B}\end{array} \\
\text { Avoirs/Eng. }\end{array}$} \\
\hline & \multicolumn{3}{|c|}{ En millions de francs } & \\
\hline Union européenne & 471716 & 416495 & 55221 & 1,13 \\
\hline Allemagne & 66622 & 68214 & -1592 & 0,98 \\
\hline Autriche & 9237 & 5333 & 3904 & 1,73 \\
\hline Belgique & 18427 & 18389 & 38 & 1,00 \\
\hline Danemark & 4827 & 2307 & 2520 & 2,09 \\
\hline Espagne & 8973 & 10678 & -1705 & 0,84 \\
\hline Finlande & 2088 & 577 & 1511 & 3,62 \\
\hline France & 43184 & 41946 & 1238 & 1,03 \\
\hline Grèce & 2693 & 4990 & -2297 & 0,54 \\
\hline Irlande & 3440 & 3877 & -437 & 0,89 \\
\hline Italie & 24115 & 31413 & -7298 & 0,77 \\
\hline Luxembourg & 16472 & 32587 & -16115 & 0,51 \\
\hline Pays-Bas & 21315 & 18278 & 3037 & 1,17 \\
\hline Portugal & 1981 & 3116 & -1135 & 0,64 \\
\hline Royaume-Uni & 241000 & 169867 & 71133 & 1,42 \\
\hline Suède & 7342 & 4923 & 2419 & 1,49 \\
\hline AELE & 3416 & 431 & 2985 & 7,93 \\
\hline Islande & 168 & 44 & 124 & 3,82 \\
\hline Norvège & 3248 & 387 & 2861 & 8,39 \\
\hline Reste de l'Europe & 23668 & 72178 & -48510 & 0,33 \\
\hline Pologne & 361 & 352 & 9 & 1,03 \\
\hline Fédération de Russie & 487 & 1528 & -1041 & 0,32 \\
\hline Turquie & 3491 & 4325 & -834 & 0,81 \\
\hline Autres pays & 19329 & 65973 & -46644 & 0,29 \\
\hline Amérique du Nord & 466182 & 299288 & 166894 & 1,56 \\
\hline Canada & 13431 & 13547 & -116 & 0,99 \\
\hline $\begin{array}{c}\text { Etats-Unis } \\
\end{array}$ & 452751 & 285741 & 167010 & 1,58 \\
\hline Région des Caraïbes & 44015 & 84677 & -40662 & 0,52 \\
\hline Bahamas & 5991 & 16636 & -10645 & 0,36 \\
\hline Iles Caïmanes & 13875 & 20089 & -6214 & 0,69 \\
\hline Antilles néerlandaises & 1770 & 2754 & -984 & 0,64 \\
\hline Panama & 3782 & 12873 & -9091 & 0,29 \\
\hline Antilles britanniques & 12370 & 25579 & -13209 & 0,48 \\
\hline Autres pays & 6227 & 6746 & -519 & 0,92 \\
\hline Amérique latine & 10749 & 22596 & -11847 & 0,48 \\
\hline Argentine & 2614 & 6738 & -4124 & 0,39 \\
\hline Brésil & 1327 & 7048 & -5721 & 0,19 \\
\hline Mexique & 2024 & 3663 & -1639 & 0,55 \\
\hline Autres pays & 4784 & 5147 & -363 & 0,93 \\
\hline
\end{tabular}




\subsection{Aperçu des relations financières avec le monde (Suisse)}

\section{B. Avoirs et engagements à l'étranger des banques établies en Suisse ${ }^{1}$}

\section{Avoirs et engagements figurant aux bilans ${ }^{2}$}

Situation au 31 décembre 2000

\begin{tabular}{|c|c|c|c|c|}
\hline \multirow[t]{2}{*}{ Pays } & $\begin{array}{c}\text { Avoirs } \\
\text { A }\end{array}$ & $\begin{array}{c}\text { Engagem. } \\
\text { B }\end{array}$ & $\begin{array}{c}\text { Solde } \\
\text { C=A-B }\end{array}$ & \multirow{2}{*}{ 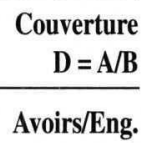 } \\
\hline & \multicolumn{3}{|c|}{ En millions de francs } & \\
\hline Moyen-Orient & 16085 & 45463 & -29378 & 0,35 \\
\hline Egypte & 1172 & 2233 & -1061 & 0,52 \\
\hline Israël & 1405 & 5715 & -4310 & 0,25 \\
\hline Arabie Saoudite & 4800 & 8686 & -3886 & 0,55 \\
\hline Autres pays & 8708 & 28829 & -20121 & 0,30 \\
\hline Afrique & 5549 & 8532 & -2983 & 0,65 \\
\hline Algérie & 64 & 228 & -164 & 0,28 \\
\hline Libéria & 1786 & 1711 & 75 & 1,04 \\
\hline Nigéria & 100 & 400 & -300 & 0,25 \\
\hline Afrique du Sud & 1529 & 2423 & -894 & 0,63 \\
\hline Autres pays & 2070 & 3770 & -1700 & 0,55 \\
\hline Asie, Océanie & 122032 & 128850 & -6818 & 0,95 \\
\hline Chine (Rép. Populaire) & 926 & 4288 & -3362 & 0,22 \\
\hline Hongkong & 5486 & 20512 & -15026 & 0,27 \\
\hline Inde & 1128 & 1509 & -381 & 0,75 \\
\hline Japon & 94346 & 73755 & 20591 & 1,28 \\
\hline Corée du Sud & 2185 & 1621 & 564 & 1,35 \\
\hline Singapour & 11111 & 10383 & 728 & 1,07 \\
\hline Autres pays & 6850 & 16782 & -9932 & 0,41 \\
\hline Australie/Nouvelle Zélande & 11644 & 6964 & 4680 & 1,67 \\
\hline Australie & 10615 & 6307 & 4308 & 1,68 \\
\hline $\begin{array}{c}\text { Nouvelle-Zélande } \\
\end{array}$ & 1029 & 657 & 372 & 1,57 \\
\hline Autres pays & 3 & 5 & -2 & 0,60 \\
\hline Total $^{3}$ & 1175054 & 1085481 & 89573 & 1,08 \\
\hline \multicolumn{5}{|l|}{ Sous-totaux } \\
\hline Pays industr. à économie de marc & 1067481 & 864786 & 202695 & 1,23 \\
\hline Pays en développement ${ }^{5}$ & 107573 & 220695 & -113122 & 0,49 \\
\hline Moyen-Orient (sans Egypte) & 14913 & 43230 & -28317 & 0,34 \\
\hline Région des Caraïbes & 44015 & 84677 & -40662 & 0,52 \\
\hline Ensemble des autres pays & 48645 & 92788 & -44143 & 0,52 \\
\hline
\end{tabular}

Voir notes et source à la page 343. 


\subsection{Aperçu des relations financières avec le monde}

B. Avoirs et engagements à l'étranger des banques établies en Suisse ${ }^{1}$

\section{Avoirs et engagements à titre fiduciaire ${ }^{2}$} Situation au 31 décembre 2000

\begin{tabular}{|c|c|c|c|c|}
\hline \multirow[t]{2}{*}{ Pays } & $\begin{array}{c}\text { Avoirs } \\
\text { A }\end{array}$ & $\begin{array}{c}\text { Engagem. } \\
\text { B }\end{array}$ & $\begin{array}{l}\text { Solde } \\
\mathrm{C}=\mathrm{A}-\mathrm{B}\end{array}$ & \multirow{2}{*}{$\begin{array}{r}\begin{array}{r}\text { Couverture } \\
\mathrm{D}=\mathrm{A} / \mathrm{B}\end{array} \\
\text { Avoirs/Eng. }\end{array}$} \\
\hline & \multicolumn{3}{|c|}{ En millions de francs } & \\
\hline Union Européenne & 320719 & 90064 & 230655 & 3,56 \\
\hline $\begin{array}{l}\text { Allemagne } \\
\end{array}$ & 15818 & 9711 & 6107 & 1,63 \\
\hline Autriche & 976 & 1815 & -839 & 0,54 \\
\hline Belgique & 52000 & 7312 & 44688 & 7,11 \\
\hline Danemark & 595 & 129 & 466 & 4,61 \\
\hline Espagne & 4131 & 7461 & -3330 & 0,55 \\
\hline Finlande & 18 & 174 & -156 & 0,10 \\
\hline France & 44524 & 13016 & 31508 & 3,42 \\
\hline Grèce & 12 & 5649 & -5637 & 0,00 \\
\hline Irlande & 5214 & 545 & 4669 & 9,57 \\
\hline Italie & 1216 & 16866 & -15650 & 0,07 \\
\hline Luxembourg & 68283 & 2205 & 66078 & 30,97 \\
\hline Pays-Bas & 63863 & 5514 & 58349 & 11,58 \\
\hline Portugal & 19 & 1836 & -1817 & 0,01 \\
\hline Royaume-Uni & 63772 & 17065 & 46707 & 3,74 \\
\hline Suède & 278 & 766 & -488 & 0,36 \\
\hline AELE & 7 & 220 & -213 & 0,03 \\
\hline Islande & 0 & 9 & -9 & 0,00 \\
\hline Norvège & 7 & 211 & -204 & 0,03 \\
\hline Reste de l'Europe & 46813 & 31263 & 15550 & 1,50 \\
\hline Gibraltar & 0 & 3331 & -3331 & 0,00 \\
\hline Guernesey & 20198 & 2430 & 17768 & 8,31 \\
\hline Ile de Man & 4574 & 5812 & -1238 & 0,79 \\
\hline Jersey & 19105 & 4200 & 14905 & 4,55 \\
\hline Monaco & 894 & 2581 & -1687 & 0,35 \\
\hline Pologne & 8 & 215 & -207 & 0,04 \\
\hline Fédération de Russie & 100 & 3507 & -3407 & 0,03 \\
\hline Ukraine & 0 & 345 & -345 & 0,00 \\
\hline Turquie & 1525 & 5314 & -3789 & 0,29 \\
\hline Autres pays & 409 & 3528 & -3119 & 0,12 \\
\hline Amérique du Nord & 1487 & 10301 & -8814 & 0,14 \\
\hline Canada & 803 & 2684 & -1881 & 0,30 \\
\hline Etats-Unis & 684 & 7617 & -6933 & 0,09 \\
\hline Région des Caraïbes & 9164 & 81275 & -72111 & 0,11 \\
\hline Bahamas & 860 & 8063 & -7203 & 0,11 \\
\hline Bermudes & 0 & 1838 & -1838 & 0,00 \\
\hline Iles Caïmanes & 7294 & 9049 & -1755 & $\overline{0,81}$ \\
\hline Antilles néerlandaises & 126 & 2672 & -2546 & 0,05 \\
\hline Panama & 75 & 19829 & -19754 & 0,00 \\
\hline Antilles britanniques & 785 & 37650 & -36865 & 0,02 \\
\hline Autres pays & 24 & 2174 & -2150 & $\overline{0,01}$ \\
\hline
\end{tabular}




\subsection{Aperçu des relations financières avec le monde}

\section{B. Avoirs et engagements à l'étranger des banques établies en Suisse}

2. Avoirs et engagements à titre fiduciaire ${ }^{2}$ Situation au 31 décembre 2000

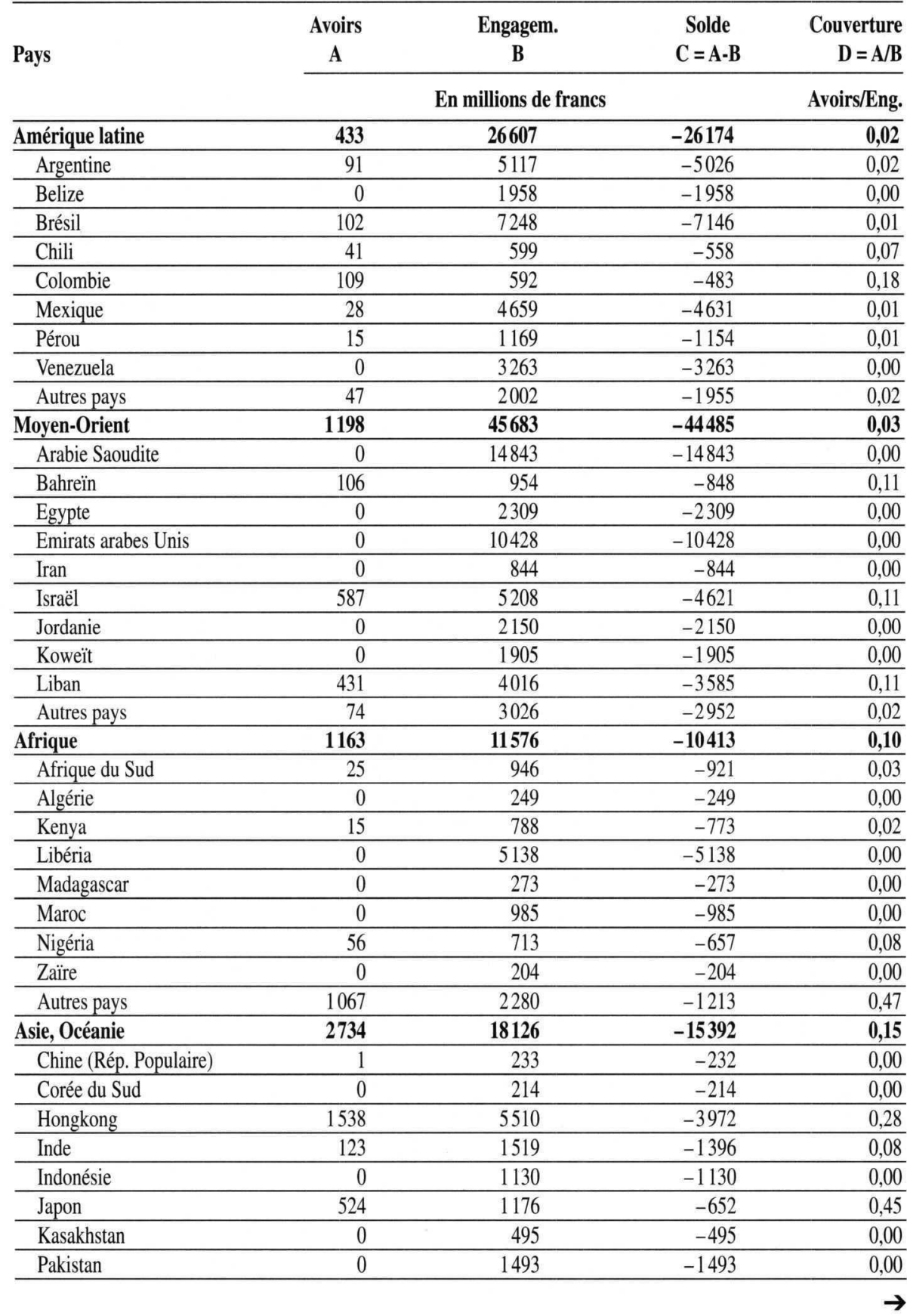




\subsection{Aperçu des relations financières avec le monde}

\section{B. Avoirs et engagements à l'étranger des banques établies en Suisse ${ }^{1}$}

\section{Avoirs et engagements à titre fiduciaire ${ }^{2}$ Situation au 31 décembre 2000}

\begin{tabular}{|c|c|c|c|c|}
\hline \multirow[t]{2}{*}{ Pays } & $\begin{array}{c}\text { Avoirs } \\
\text { A }\end{array}$ & $\begin{array}{c}\text { Engagem. } \\
\text { B }\end{array}$ & $\begin{array}{c}\text { Solde } \\
C=A-B\end{array}$ & $\begin{array}{r}\text { Couverture } \\
\mathrm{D}=\mathrm{A} / \mathrm{B}\end{array}$ \\
\hline & \multicolumn{3}{|c|}{ En millions de francs } & Avoirs/Eng. \\
\hline Philippines & 0 & 642 & -642 & 0,00 \\
\hline Singapour & 432 & 1573 & -1141 & 0,27 \\
\hline Taiwan & 0 & 2003 & -2003 & 0,00 \\
\hline Autres pays & 116 & 2138 & -2022 & 0,05 \\
\hline Australie/Nouvelle Zélande & 83 & 1574 & -1491 & 0,05 \\
\hline Australie & 37 & 906 & -869 & 0,04 \\
\hline Nouvelle-Zélande & 46 & 668 & -622 & 0,07 \\
\hline Autres pays & 13 & 230 & -217 & 0,06 \\
\hline Total $^{3}$ & 383816 & 316920 & 66896 & 1,21 \\
\hline \multicolumn{5}{|l|}{ Sous-totaux } \\
\hline Pays industr. à économie de marchét & 368108 & 129284 & 238824 & 2,85 \\
\hline Pays en développement ${ }^{5}$ & 15708 & 187636 & -171928 & 0,08 \\
\hline Moyen-Orient (sans Egypte) & 1198 & 43374 & -42176 & 0,03 \\
\hline Région des Caraïbes & 9164 & 81275 & -72111 & 0,11 \\
\hline Ensemble des autres pays & 5346 & 62987 & -57641 & 0,08 \\
\hline
\end{tabular}

Source: Banque nationale suisse, Les banques suisses 2000 , tableau V p. 40 ; tableau VI p. 41 , tableau 32.0 (pp. A124 à A135) et tableau 38.o (p. 153), août 2001.

Remarques: La Banque nationale suisse publie une statistique des avoirs et des engagements à l'étranger des banques établies en Suisse. Elle distingue les opérations que les banques effectuent pour leur propre compte (inscrites au bilan, voir tableau 2.3.B.1) des transactions réalisées pour le compte et aux risques des clients (opérations fiduciaires, tableau 2.3.B.2). La statistique montre que les avoirs des banques suisses dans les pays en développement sont inférieurs à leurs engagements envers ces pays. En 1999, le taux de couverture a atteint $60 \%$

\section{Notes des tableaux 2.3.B.1 et 2.3.B.2}

1. Selon les comptes communiqués par 122 banques qui effectuent des opérations internationales. Les avoirs et les engagements des comptoirs (mais pas ceux des filiales) à l'étranger sont compris dans la statistique.

2. Une partie seulement des opérations bancaires avec l'étranger se reflète dans les bilans : les transactions que les banques effectuent pour leur propre compte. Par contre, les services que les banques rendent à la clientèle, comme la gestion de fortune, les opérations en bourse, les émissions, les opérations fiduciaires, le commerce des devises, l'ouverture d'accréditifs, les cautionnements, etc., n'apparaissent pas dans les bilans.

3. Sans les métaux précieux.

4. Y compris les pays PECO/CEI et le Japon; sans la Turquie.

5. Soit les régions: Afrique, Amérique latine, Asie (sans le Japon), Caraibes, Moyen-Orient, ainsi que la Turquie et la rubrique Autres pays. 


\subsection{Investissements directs suisses à l'étranger, en millions de francs}

\section{A. Répartition géographique des flux et stocks d'investissements par année}

\begin{tabular}{|c|c|c|c|c|c|c|c|}
\hline \multirow{2}{*}{ Pays } & \multicolumn{3}{|c|}{ Flux d'investissements } & \multicolumn{4}{|c|}{ Stock d'investissements } \\
\hline & 1998 & $1999^{r}$ & $2000^{p}$ & 1998 & $1999^{r}$ & $2000^{p}$ & $\begin{array}{r}\text { Variation } \\
\text { 00/99en \% } \\
\end{array}$ \\
\hline Pays industrialisés & 16090 & 38128 & 61247 & 197861 & 241873 & 294871 & 21,9 \\
\hline Union européenne & 10154 & 21359 & 25392 & 119025 & 149983 & 178679 & 19,1 \\
\hline Belgique & 349 & 1179 & 5605 & 3396 & 4924 & 10381 & 110,8 \\
\hline Danemark & -31 & 280 & -84 & 632 & 1187 & 1076 & $-9,4$ \\
\hline Allemagne & 889 & 5799 & -1207 & 18901 & 26784 & 26110 & $-2,5$ \\
\hline Finlande & 224 & 126 & 184 & 1387 & 1794 & 1846 & 2,9 \\
\hline France & 333 & 827 & -1568 & 13640 & 16594 & 15685 & $-5,5$ \\
\hline Grèce & 205 & 179 & 352 & 398 & 2317 & 2024 & $-12,6$ \\
\hline Irlande & -54 & 5446 & 1319 & 5478 & 10282 & 12380 & 20,4 \\
\hline Italie & -197 & 192 & -265 & 7607 & 9766 & 9715 & $-0,5$ \\
\hline Luxembourg & 1651 & -47 & 1118 & 6005 & 7849 & 10777 & 37,3 \\
\hline Pays-Bas & 1003 & 1716 & 2747 & 16826 & 18869 & 20657 & 9,5 \\
\hline Autriche & 344 & 515 & 114 & 3123 & 4434 & 4707 & 6,2 \\
\hline Portugal & -69 & 561 & 242 & 995 & 1747 & 1907 & 9,2 \\
\hline Suède & 844 & -656 & 382 & 3420 & 2005 & 2141 & 6,8 \\
\hline Espagne & 377 & -545 & 5 & 4651 & 5574 & 5299 & $-4,9$ \\
\hline Royaume-Uni ${ }^{1}$ & 4287 & 5788 & 16450 & 32567 & 35858 & 53976 & 50,5 \\
\hline AELE & 93 & 362 & 143 & 1485 & 3129 & 3238 & 3,5 \\
\hline Europe centrale et orientale & 1175 & 1174 & 1098 & 4462 & 6156 & 6528 & 6,0 \\
\hline dont Croatie & 70 & -44 & 16 & 117 & 159 & 217 & 36,5 \\
\hline Pologne & 208 & 473 & 587 & 1062 & 1667 & 2168 & 30,1 \\
\hline Fédération de Russie & 335 & 599 & -10 & 748 & 1197 & 744 & $-37,8$ \\
\hline République tchèque & 356 & -64 & 195 & 1648 & 1625 & 1762 & 8,4 \\
\hline Slovaquie & 21 & 38 & 12 & 103 & 157 & 146 & $-7,0$ \\
\hline Hongrie & -76 & 100 & 75 & 328 & 868 & 1000 & 15,2 \\
\hline Reste de l'Europe & -449 & 744 & 569 & 1082 & 2727 & 2753 & 1,0 \\
\hline dont Turquie & 296 & -95 & 244 & 725 & 1019 & 1041 & 2,2 \\
\hline Amérique du Nord & 4458 & 13640 & 33973 & 63934 & 68986 & 94063 & 36,4 \\
\hline Canada & 1232 & 83 & 43 & 6276 & 3511 & 5275 & 50,2 \\
\hline Etats-Unis & 3226 & 13557 & 33930 & 57658 & 65475 & 88788 & 35,6 \\
\hline $\begin{array}{l}\text { Autres pays industrialisés } \\
\text { non européens }\end{array}$ & 845 & 851 & 73 & 7874 & 10892 & 9610 & $-11,8$ \\
\hline Australie & 572 & 322 & -263 & 3147 & 3923 & 3131 & $-20,2$ \\
\hline Japon & 124 & 628 & 318 & 3730 & 5187 & 4692 & $-9,5$ \\
\hline Nouvelle-Zélande & 11 & -215 & -1 & 125 & 451 & 466 & 3,3 \\
\hline Afrique du Sud & 138 & 116 & 19 & 872 & 1331 & 1322 & $-0,7$ \\
\hline Pays nouv. ${ }^{\text {ment }}$ industrialisés & 6932 & 6244 & 1441 & 25711 & 31929 & 33015 & 3,4 \\
\hline
\end{tabular}

${ }^{\mathrm{r}}$ chiffres révisés.

${ }^{\mathrm{p}}$ chiffres provisoires.

1. Y compris Guernesey, Jersey et l'île de Man. 


\subsection{Investissements directs suisses à l'étranger, en millions de franc (suite)}

\section{A. Répartition géographique des flux et stocks d'investissements par année}

\begin{tabular}{|c|c|c|c|c|c|c|c|c|}
\hline \multirow{2}{*}{ Pays } & & \multicolumn{3}{|c|}{ Flux d'investissements } & \multicolumn{4}{|c|}{ Stock d'investissements } \\
\hline & & 1998 & $1999^{r}$ & $2000^{p}$ & 1998 & $1999^{r}$ & $2000^{p}$ & $\begin{array}{r}\text { Variation } \\
\text { 00/99en \% }\end{array}$ \\
\hline \multicolumn{2}{|l|}{ Asie } & 5690 & 4949 & 292 & 16804 & 20805 & 20819 & 0,1 \\
\hline & Hongkong & 447 & 665 & -60 & 2064 & 2316 & 2647 & 14,3 \\
\hline & Corée (Sud) & 283 & 179 & 186 & 692 & 997 & 1049 & 5,2 \\
\hline & Malaisie & 102 & 227 & -28 & 901 & 937 & 1000 & 6,7 \\
\hline & Philippines & 1169 & 57 & 295 & 1281 & 1433 & 1638 & 14,3 \\
\hline & Singapour & 3173 & 3811 & -287 & 10755 & 13779 & 13105 & $-4,9$ \\
\hline & Taïwan & 121 & 92 & 158 & 480 & 631 & 681 & 7,9 \\
\hline & Thaïlande & 395 & -82 & 28 & 631 & 712 & 699 & $-1,8$ \\
\hline \multicolumn{2}{|c|}{ Amérique centrale et du Sud } & 1243 & 1295 & 1148 & 8908 & 11124 & 12197 & 9,6 \\
\hline \multicolumn{2}{|c|}{ Argentine } & 321 & -103 & 48 & 1085 & 1317 & 1705 & 29,5 \\
\hline \multicolumn{2}{|c|}{ Brésil } & 446 & 844 & 220 & 4375 & 5072 & 5333 & 5,1 \\
\hline \multicolumn{2}{|r|}{ Chili } & -178 & 17 & -24 & 686 & 828 & 791 & $-4,5$ \\
\hline & Mexique & 654 & 537 & 904 & 2762 & 3907 & 4368 & 11,8 \\
\hline \multicolumn{2}{|c|}{ Pays en développement } & 4187 & 5614 & 9338 & 30024 & 37457 & 44680 & 19,3 \\
\hline \multicolumn{2}{|c|}{ Asie } & 441 & -21 & 200 & 3441 & 4116 & 4668 & 13,4 \\
\hline \multirow[t]{8}{*}{ dont } & Chine (Rép. pop.) & 123 & -143 & 206 & 1362 & 1403 & 1530 & 9,1 \\
\hline & Inde & 29 & -59 & -33 & 448 & 529 & 414 & $-21,7$ \\
\hline & Indonésie & 90 & 10 & -57 & 297 & 391 & 445 & 13,8 \\
\hline & Liban & -2 & -13 & -144 & 120 & 120 & 104 & $-13,3$ \\
\hline & Pakistan & -17 & 7 & 60 & 118 & 192 & 211 & 9,9 \\
\hline & Arabie Saoudite & -2 & 60 & -84 & 253 & 323 & 332 & 2,8 \\
\hline & Emirats arabes unis & 11 & 19 & 69 & 114 & 143 & 206 & 44,1 \\
\hline & Vietnam & -2 & -18 & -32 & 128 & 128 & 112 & $-12,5$ \\
\hline \multicolumn{2}{|c|}{ Amérique centrale et du Sud } & 3645 & 5343 & 8982 & 24964 & 30721 & 36799 & 19,8 \\
\hline \multirow[t]{8}{*}{ dont } & Costa Rica & -26 & 0 & -17 & 138 & 180 & 130 & $-27,8$ \\
\hline & Equateur & 10 & 20 & 24 & 299 & 376 & 441 & 17,3 \\
\hline & Guatemala & -4 & 22 & 97 & 118 & 145 & 87 & $-40,0$ \\
\hline & Colombie & 387 & 115 & -110 & 974 & 1166 & 1050 & $-9,9$ \\
\hline & Pérou & -25 & 47 & 57 & 192 & 261 & 314 & 20,3 \\
\hline & Uruguay & 284 & 291 & 302 & 307 & 447 & 418 & $-6,5$ \\
\hline & Venezuela & 61 & 11 & 75 & 621 & 696 & 744 & 6,9 \\
\hline & Centres financ. off-shore ${ }^{2}$ & 2991 & 4703 & 8799 & 22098 & 26993 & 32540 & 20,5 \\
\hline \multicolumn{2}{|c|}{ Afrique } & 102 & 293 & 155 & 1619 & 2621 & 3213 & 22,6 \\
\hline \multirow[t]{4}{*}{ dont } & Egypte & 57 & 93 & 10 & 354 & 472 & 609 & 29,0 \\
\hline & Côte d'Ivoire & 51 & -26 & -33 & 101 & 114 & 115 & 0,9 \\
\hline & Maroc & -12 & 2 & -30 & 330 & 318 & 294 & $-7,5$ \\
\hline & Nigéria & 5 & -7 & 2 & 26 & 35 & 29 & $-17,1$ \\
\hline \multicolumn{2}{|c|}{ Total Monde } & 27209 & 49986 & 72026 & 253596 & 311258 & 372566 & 19,7 \\
\hline
\end{tabular}

Source: Banque nationale suisse, Bulletin trimestriel 4/2001, BNS, 2001. Sur Internet <www.snb.ch>.

2. Centre financiers off-shore: Bahamas, Barbade, Bermudes, îles Vierges britanniques, Jamaïque, îles Caïmanes, Montserrat, Antilles néerlandaises, Panama, St Kitts et Nevis. 


\subsection{Personnel dans les entreprises suisses à l'étranger}

\section{B. Effectif du personnel en fin d'année}

\begin{tabular}{|c|c|c|c|c|}
\hline \multirow{2}{*}{ Pays } & \multicolumn{4}{|c|}{ Nombre d'employés } \\
\hline & 1998 & 1999 & $2000^{p}$ & $\begin{array}{r}\text { Variation } \\
\text { 00/99en \% }\end{array}$ \\
\hline Pays industrialisés & 1219740 & 1241472 & 1322526 & 6,5 \\
\hline Union européenne & 778499 & 780845 & 793908 & 1,7 \\
\hline Belgique & 25125 & 25899 & 29370 & 13,4 \\
\hline Danemark & 11356 & 11223 & 10813 & $-3,7$ \\
\hline Allemagne & 268107 & 249165 & 251625 & 1,0 \\
\hline Finlande & 13793 & 13607 & 14397 & 5,8 \\
\hline France & 121377 & 132421 & 128885 & $-2,7$ \\
\hline Grèce & 8397 & 8392 & 8850 & 5,5 \\
\hline Irlande & 4347 & 5332 & 5081 & $-4,7$ \\
\hline Italie & 56795 & 58581 & 61870 & 5,6 \\
\hline Luxembourg & 1852 & 2487 & 2547 & 2,4 \\
\hline Pays-Bas & 32380 & 31441 & 29824 & $-5,1$ \\
\hline Autriche & 27775 & 33001 & 34713 & 5,2 \\
\hline Portugal & 9291 & 10172 & 9750 & $-4,1$ \\
\hline Suède & 37057 & 32572 & 30053 & $-7,7$ \\
\hline Espagne & 45568 & 44383 & 45782 & 3,2 \\
\hline Royaume-Unil & 115281 & 122172 & 130350 & 6,7 \\
\hline AELE & 12631 & 12621 & 12756 & 1,1 \\
\hline Europe centrale et orientale & 77213 & 73059 & 82416 & 12,8 \\
\hline dont Croatie & 2540 & 2170 & 2853 & 31,5 \\
\hline Pologne & 23193 & 19893 & 22753 & 14,4 \\
\hline Fédération de Russie & 8836 & 9692 & 10825 & 11,7 \\
\hline République tchèque & 18768 & 15982 & 16829 & 5,3 \\
\hline Slovaquie & 3185 & 3905 & 3616 & $-7,4$ \\
\hline Hongrie & 9692 & 9581 & 11396 & 18,9 \\
\hline Reste de l'Europe & 9750 & 10858 & 11986 & 10,4 \\
\hline dont Turquie & 9129 & 10228 & 10616 & 3,8 \\
\hline Amérique du Nord & 268176 & 291243 & 344593 & 18,3 \\
\hline Canada & 26744 & 28011 & 34680 & 23,8 \\
\hline Etats-Unis & 241432 & 263232 & 309913 & 17,7 \\
\hline $\begin{array}{l}\text { Autre pays industrialisés } \\
\text { non européens }\end{array}$ & 73471 & 72847 & 76867 & 5,5 \\
\hline Australie & 27338 & 24651 & 26542 & 7,7 \\
\hline Japon & 21749 & 24060 & 27015 & 12,3 \\
\hline Nouvelle-Zélande & 4006 & 3223 & 3342 & 3,7 \\
\hline Afrique du Sud & 20378 & 20913 & 19968 & $-4,5$ \\
\hline Pays nouvellement industrialisés & 223014 & 224915 & 233689 & 3,9 \\
\hline
\end{tabular}

Source: Banque nationale suisse, bulletin trimestriel 4/2001, Zurich, BNS, 2001. www.snb.ch

1. Y compris Guernesey, Jersey et l'île de Man. 


\subsection{Personnel dans les entreprises suisses à l'étranger (suite) \\ B. Effectif du personnel en fin d'année}

\begin{tabular}{|c|c|c|c|c|c|}
\hline \multirow{2}{*}{\multicolumn{2}{|c|}{ Pays }} & \multicolumn{4}{|c|}{ Nombre d'employés } \\
\hline & & \multirow{2}{*}{$\begin{array}{r}1998 \\
107690\end{array}$} & \multirow{2}{*}{$\begin{array}{c}1999^{\mathrm{r}} \\
102073\end{array}$} & \multirow{2}{*}{$\begin{array}{r}2000^{p} \\
111927\end{array}$} & \multirow{2}{*}{$\begin{array}{r}\begin{array}{r}\text { Variation } \\
\text { 00/99en \% }\end{array} \\
9,7\end{array}$} \\
\hline Asie & & & & & \\
\hline & Hongkong & 14319 & 14016 & 14828 & 5,8 \\
\hline & Corée du Sud & 4007 & 4716 & 5284 & 12,0 \\
\hline & Malaisie & 15982 & 13035 & 14665 & 12,5 \\
\hline & Philippines & 13293 & 13050 & 13172 & 0,9 \\
\hline & Singapour & 18945 & 19137 & 19706 & 3,0 \\
\hline & Tä̈wan & 10424 & 9887 & 9984 & 1,0 \\
\hline & Thaïlande & 30720 & 28232 & 34288 & 21,5 \\
\hline Amér & que centrale et du Sud & 115325 & 122842 & 121763 & $-0,9$ \\
\hline & Argentine & 11961 & 12995 & 13303 & 2,4 \\
\hline & Brésil & 67552 & 72322 & 71541 & $-1,1$ \\
\hline & Chili & 10622 & 9819 & 9497 & $-3,3$ \\
\hline & Mexique & 25190 & 27706 & 27422 & $-1,0$ \\
\hline Pays & $\mathrm{n}$ développement & 169191 & 174569 & 179582 & 2,9 \\
\hline Asie & & 89994 & 94790 & 100801 & 6,3 \\
\hline \multirow[t]{8}{*}{ dont } & Chine (Rép. populaire) & 32795 & 37457 & 40257 & 7,5 \\
\hline & Inde & 21604 & 19443 & 20326 & 4,5 \\
\hline & Indonésie & 10031 & 10021 & 11253 & 12,3 \\
\hline & Liban & 872 & 909 & 721 & $-20,7$ \\
\hline & Pakistan & 3521 & 4298 & 4848 & 12,8 \\
\hline & Arabie Saoudite & 3255 & 2866 & 2888 & 0,8 \\
\hline & Emirats arabes unis & 1427 & 1190 & 1051 & $-11,7$ \\
\hline & Vietnam & 4567 & 5086 & 5198 & 2,2 \\
\hline \multicolumn{2}{|c|}{ Amérique centrale et du Sud } & 36376 & 41346 & 38329 & $-7,3$ \\
\hline \multirow[t]{8}{*}{ dont } & Costa Rica & 3032 & 2856 & 2313 & $-19,0$ \\
\hline & Equateur & 2911 & 3418 & 3302 & $-3,4$ \\
\hline & Guatemala & 1481 & 1539 & 1625 & 5,6 \\
\hline & Colombie & 7292 & 8479 & 8432 & $-0,6$ \\
\hline & Pérou & 3736 & 3895 & 3738 & $-4,0$ \\
\hline & Uruguay & 869 & 858 & 910 & 6,1 \\
\hline & Venezuela & 8092 & 8493 & 7739 & $-8,9$ \\
\hline & Centres financiers off-shore ${ }^{2}$ & 5743 & 6122 & 5064 & $-17,3$ \\
\hline \multicolumn{2}{|c|}{ Afrique } & 42821 & 38433 & 40451 & 5,3 \\
\hline \multirow[t]{4}{*}{ dont } & Egypte & 9793 & 8428 & 8422 & $-0,1$ \\
\hline & Côte d'Ivoire & 2517 & 2217 & 3021 & 36,3 \\
\hline & Maroc & 3652 & 3489 & 3477 & $-0,3$ \\
\hline & Nigéria & 6047 & 5505 & 6745 & 22,5 \\
\hline \multicolumn{2}{|c|}{ Total Monde } & 1611945 & 1640956 & 1735795 & 5,8 \\
\hline
\end{tabular}

2. Centre financiers off-shore: Bahamas, Barbade, Bermudes, îles Vierges britanniques, Jamaïque, îles Caïmanes, Montserrat, Antilles néerlandaises, Panama, St Kitts et Nevis. 


\section{Remarques pour les tableaux 2.4.A et 2.4.B}

\section{Définition de base :}

Celui qui effectue un investissement direct veut influencer directement et durablement sur l'activité d'une entreprise (en créant une filiale ou succursale à l'étranger ou en participant à $10 \%$ au moins au capital d'une entreprise à l'étranger).

\section{Méthode de recueil des données :}

La Banque nationale suisse recueille chaque année les données sur les investissements directs suisses à l'étranger. L'enquête est obligatoire pour toutes les entreprises ayant des capitaux d'investissement direct de plus de 10 millions de francs.

Les entreprises fournissent des informations:

口 sur les flux financiers portant sur des participations aux fonds propres (créations de filiales, acquisitions, ventes, augmentations de capital). Le signe moins (-) indique un rapatriement de capitaux vers la Suisse. (tableau 2.4.A partie gauche).

- sur le volume du capital investi au 31 décembre (participations aux fonds propres et crédits au sein du groupe), (tableau 2.4.A. partie droite). Le volume (stock) d'investissement à l'étranger à la fin d'une année ne correspond pas forcément au stock de l'année précédente additionné au flux de l'année, car les variations du volume peuvent aussi provenir de facteurs n'apparaissant pas dans les flux de capitaux (acquisitions à l'étranger financées par le bénéfice réinvesti de filiales à l'étranger, évolution des cours du change, modifications dans les méthodes comptables, etc.).

口 sur l'effectif de leur personnel dans les filiales et succursales à l'étranger (tableau 2.4.B).

\section{Répartition des investissements par pays :}

Le pays mentionné dans les tableaux est dans la mesure du possible le pays bénéficiaire final des investissements directs étrangers de la Suisse. Ce principe ne peut toutefois pas toujours être appliqué (dans le cas par exemple des pays des Caraïbes, plaques tournantes de mouvements internationaux de capitaux, ou dans le cas des paradis fiscaux).

La classification des pays diffère de celle des tableaux précédents. Les totaux «pays en développement » des tableaux 2.4 comprennent les pays en développement avancés qui ne devraient, pour le Comité d'aide au développement (CAD) de l'OCDE, plus être compris dans cette liste des pays en développement. Les pays en développement européens (Turquie et Etats de l'ex Yougoslavie) sont dans les tableaux 2.4 compris dans la liste des pays industrialisés.

Voir aussi les tableaux 4.1. et 4.2. pour les investissements classés selon le CAD (liste des pays en développement et en transition à la fin de cet Annuaire).

Le total des flux d'investissement direct s'est élevé à 61,2 milliards de francs en l'an 2000. Selon les tableaux de la partie 4, seuls 1,9 milliard de francs a été investi dans les pays en développement. 9,8 milliards ont été investis dans les pays en développement plus avancés et 0,9 milliard dans les pays d'Europe centrale et orientale/Nouveaux Etats indépendants. 